\title{
Study of the Soret effect in mixed conductors by the measurement of ionic and electronic thermopower
}

\author{
J. Janek* , C. Korte \\ Institut für Physikalische Chemie und Elektrochemie und Sonderforschungsbereich 173 der Universität Hannover, Callinstrasse 3-3A. \\ 30167 Hannover. Germany
}

\begin{abstract}
The redistribution of a mobile component in a mixed conducting compound $\mathrm{A}_{1-\delta} \mathrm{X}_{r}$ in a temperature gradient can be studied by means of time-dependent measurements of either the ionic or the electronic thermovoltage. The reduced heat of transport of the mobile component $\left(Q_{\mathrm{A}}^{*}\right.$ or $\left.Q_{X}^{*}\right)$ results directly from the difference of the partial thermovoltages at $t \rightarrow 0$ and $t \rightarrow \infty$ : For materials with predominant ionic conductivity, the electronic thermopower has to be measured; for materials with predominant electronic conductivity, the ionic thermopower has to be measured. Experimental examples are presented.
\end{abstract}

Keywords: Mixed conductors; Soret effect; Heat of transport; Ionic thermopower; Electronic thermopower

\section{Introduction}

Binary ionic compounds of the type $\mathrm{A}_{1+\delta} \mathrm{X}_{v}$ generally exhibit both ionic and electronic conductivity in their magnitude, depending on the actual defect structure and the defect mobilities. In a temperature gradient, thermal diffusion of the mobile charge carriers leads to the establishment of a gradient of the metal excess (or deficit) $\delta$ according to the expression

$$
\begin{aligned}
\left(\frac{1}{\delta} \frac{\mathrm{d} \delta}{\mathrm{d} T}\right)_{j_{\mathrm{A}}=0} & =-\frac{Q_{\mathrm{A}}^{*}}{\delta \cdot T} \cdot\left(\frac{\partial \mu_{\mathrm{A}}}{\partial \delta}\right)_{T}^{-1} \\
& =-\frac{Q_{\mathrm{A}}^{*}}{R T^{2}} \cdot\left(\frac{\partial \ln a_{\mathrm{A}}}{\partial \ln \delta}\right)_{T}^{-1}
\end{aligned}
$$

\footnotetext{
${ }^{*}$ Corresponding author.
}

if the surfaces of the crystal are closed for matter exchange $\left(j_{\mathrm{A}}=0\right)$. In deriving Eq. (1), the metal component is assumed to be mobile. An equivalent relation can be formulated for anion-conducting compounds.

The (reduced ') heat of transport $Q_{\mathrm{A}}^{*}$ of the mobile component decides on the magnitude and the direction of the stationary composition gradient, besides the thermodynamic factor $\left(\partial \ln a_{\mathrm{A}} / \partial \ln \delta\right)_{T}$, with $\mu_{\mathrm{A}}$ and $a_{\mathrm{A}}$ denoting the chemical potential and the thermodynamic activity of metal $A$, respectively. Only for a few mixed conducting systems has the heat of transport of the mobile component been determined experimentally. Thus quantitative data exist for $Q_{\mathrm{Ag}}^{*}$ in the cation-conducting compounds $\alpha-\mathrm{Ag}_{2+\delta} \mathrm{S}$ [2], $\alpha-\mathrm{Ag}_{2+\delta} \mathrm{Se}[3], \beta-\mathrm{Ag}_{2+\delta} \mathrm{S}$ [4-8] and

'Only the reduced heat of transport $Q_{1}^{*}$ will be used in the following, cf. [1]. 
AgI [9], and for $Q_{0}^{*}$ in the anion-conducting compounds $(\mathrm{U}, \mathrm{Pu}) \mathrm{O}_{2+\delta}[10], \mathrm{CeO}_{2-\delta}[11],(\mathrm{Ce}, \mathrm{U}) \mathrm{O}_{2-\delta}$ [12] and $\mathrm{ZrO}_{2}\left(\mathrm{Y}_{2} \mathrm{O}_{3}\right)$ [13]. The concentration gradients which are defined by Eq. (1) are usually small, and thus, cannot be measured by conventional analytical techniques. For example, in the case of the extremely narrow $\alpha-\mathrm{Ag}_{2+\delta} \mathrm{S}$ phase, a temperature difference of $10 \mathrm{~K}$ at an average temperature of $160^{\circ} \mathrm{C}$ leads to a difference $\Delta \delta \simeq 5.4 \times 10^{-7}$ between the ends of the crystal at an average metal excess of $\delta=2.3 \times 10^{-6}$ [cf. Ref. [2]; $\left(\partial \mu_{\mathrm{Ag}} / \partial \delta\right)_{T}=1.5 \times 10^{9}$ $\left.\mathrm{J} \cdot \mathrm{mol}^{-1}, Q_{\mathrm{Ag}}^{*}=-35 \mathrm{~kJ} \cdot \mathrm{mol}^{-1}\right]$. Appearing very small in its absolute value, the demixing is appreciable in terms of the relative effect, $\partial \delta / \delta=23 \%$.

Only an electrochemical technique which measures the difference in the chemical potential $d \mu_{A}$,

$$
\begin{aligned}
\left(\frac{\mathrm{d} \mu_{\mathrm{A}}}{\mathrm{d} T}\right)_{j_{\mathrm{A}}=0} & =\left(\frac{\partial \mu_{\mathrm{A}}}{\partial T}\right)_{\delta}-\frac{Q_{\mathrm{A}}^{*}}{T} \\
& =-\left(\bar{S}_{\mathrm{A}}+\frac{Q_{\mathrm{A}}^{*}}{T}\right)
\end{aligned}
$$

that is induced by a temperature difference $d T$ in the stationary state, offers quantitative information on the component demixing in narrow range compounds. However, since the chemical potential $\mu_{\mathrm{A}}$ of the mobile component in a temperature gradient is not only changed by the component redistribution, rather more by the partial entropy $\bar{S}_{\mathrm{A}}=-\left(\partial \mu_{\mathrm{A}} / \partial T\right)_{\delta}$, independent information on the partial entropy $\bar{S}_{\mathrm{A}}$ of the metal has to be supplied for the evaluation of $Q_{\mathrm{A}}^{*}$. Alternatively, as is shown in the following, a single thermopower experiment can provide enough information for the quantitative determination of $Q_{A}^{*}$ in most mixed conductors, without the need for additional experimental information.

Thus, we analyse subsequently the formal description of the Soret effect in terms of the partial ionic and electronic thermopower. It is shown that the heat of transport of mobile components can be determined very effectively for materials with either predominant electronic or ionic conductivity. Finally, we give experimental examples for time-dependent thermopower measurements to exemplify the formal analysis. The necessary conditions for a successful evaluation of $Q_{\mathrm{A}}^{*}$ (or $Q_{\mathrm{X}}^{*}$ ) are discussed.

\section{Formal analysis}

Wagner [14] published an extensive analysis of the ionic and electronic thermopower in mixed conducting materials, including expressions for the partial thermopowers under different boundary conditions. However, the consequence for the experimental determination of heats of transport of mobile components was not pointed out explicitly, and thus is derived below.

Regarding a binary compound $\mathrm{A}_{1+\delta} \mathrm{X}_{\nu}$ with disorder in the cation (or anion) sublattice, one ionic flux $j_{\mathrm{A}^{-}}$(or the flux $j_{\mathrm{X}^{-/,-}}$of anions ${ }^{2}$ ) and the electronic flux $j_{c}$-are sufficient for the description of all matter and charge transport processes in the bulk:

$$
\begin{aligned}
& j_{\mathrm{A}^{*+}}=-L_{\mathrm{A}^{*}} \cdot\left[\nabla \tilde{\mu}_{\mathrm{A}^{*-}}+\left(\bar{S}_{\mathrm{A}^{*-}}+\frac{Q_{\mathrm{A}^{*}}^{*}}{T}\right) \nabla T\right], \\
& j_{\mathrm{e}^{-}}=-L_{\mathrm{e}^{-}} \cdot\left[\nabla \tilde{\mu}_{\mathrm{e}^{-}}+\left(\bar{S}_{\mathrm{e}^{--}}+\frac{Q_{\mathrm{e}^{-}}^{*}}{T}\right) \nabla T\right] .
\end{aligned}
$$

In Eqs. (3) and (4), the $L_{i}$ denote phenomenological transport coefficients $\left[L_{i}=\sigma_{i} /\left(z_{i} F\right)^{2}\right] . \tilde{\mu}_{i} \mathrm{de}-$ notes the electrochemical potential, $\bar{S}_{i}$ represents the partial entropy and $Q_{i}^{*}$ the (reduced) heat of transport of the charge carrier $i$. It has to be noted that the fluxes in Eqs. (3) and (4) represent macroscopic fluxes as they imply no information on the actual mobile defects. For the description of the transport experiments on the basis of a microscopic defect model, a more elaborate description had to be used $[15,16]$. In the case of coupled (thermal or chemical) diffusion of both charge carriers, one defines the metal flux $j_{\mathrm{A}} \equiv j_{\mathrm{A}^{*}+}=j_{\mathrm{e}^{-}} / z$, which is then given as

$j_{\mathrm{A}}=-L_{\mathrm{A}} \cdot\left[\nabla \mu_{\mathrm{A}}+\left(\bar{S}_{\mathrm{A}}+\frac{Q_{\mathrm{A}}^{*}}{T}\right) \nabla T\right]$.

The heat of transport $Q_{\AA}^{*}$ of metal A equals the sum of the individual heats of transport of ions and electrons, i.e. it is $Q_{\mathrm{A}}^{*}=Q_{\mathrm{A}^{*}+}^{*}+z \cdot Q_{\mathrm{e}^{-}}^{*}$ (cf. [16] for an analysis of the ionic and electronic heats of transport in terms of point defects). The chemical

\footnotetext{
For the sake of simplicity. only the case of a cation-conducting material is analysed in the following. The treatment of anionconducting materials is equivalent, and thus, only the final results are given below.
} 
potential of the metal is defined as $\mu_{\mathrm{A}}=\tilde{\mu}_{\mathrm{A}:+}+z$. $\tilde{\mu}_{\mathrm{e}^{-}}$, the entropy of metal is $\bar{S}_{\mathrm{A}^{\mathrm{B}}}=\bar{S}_{\mathrm{A}^{*}+}+z \cdot \bar{S}_{\mathrm{c}^{-}}$, and the transport coefficient of the metal is given as $L_{\mathrm{A}}=L_{\mathrm{A}^{-}} L_{\mathrm{e}^{-}} /\left(L_{\mathrm{e}^{-}}+L_{\mathrm{A}^{*-}}\right)$. The chemical potential gradient itself is a function (total differential) of the applied temperature gradient and the composition gradient:

$$
\begin{aligned}
\nabla \mu_{\mathrm{A}} & =\left(\frac{\partial \mu_{\mathrm{A}}}{\partial \delta}\right)_{T} \nabla \delta+\left(\frac{\partial \mu_{\mathrm{A}}}{\partial T}\right)_{\delta} \nabla T \\
& =\left(\frac{\partial \mu_{\mathrm{A}}}{\partial \delta}\right)_{T} \nabla \delta-\bar{S}_{\mathrm{A}} \nabla T
\end{aligned}
$$

Thus, for a material with an initially homogeneous metal distribution $(\nabla \delta=0)$, the metal flux in a temperature gradient in the very beginning is determined solely by the heat of transport, i.e. is only caused by thermal diffusion [combine Eqs. (5) and (6)]:

$j_{\mathrm{A}}=-L_{\mathrm{A}} \cdot \frac{Q_{\mathrm{A}}^{*}}{T} \nabla T \quad(\nabla \delta=0)$.

In the stationary state, the chemical potential difference, cf. Eq. (2), can be determined from measurements with suitable chemical potential probes. A corresponding experimental study of the Soret effect in $\beta-\mathrm{Ag}_{2+\delta} \mathrm{S}$ has been published recently [7]. For materials with either predominant ionic (transference number $t_{\mathrm{e}^{-}} \simeq 1$ ) or electronic $\left(t_{\mathrm{A}^{-}+} \simeq 1\right.$ ) conductivity, the chemical potential difference can also be obtained from a single thermopower experiment with partially blocking electrodes:

\subsection{Measurements with inert metal electrodes}

For a thermogalvanic cell involving a mixed conducting compound $\mathrm{A}_{1+\delta} \mathrm{X}_{v}$ with inert metal electrodes (e.g. Pt) which are impermeable for both components $\mathrm{A}$ and $\mathrm{X}$,

$(\alpha) \operatorname{Pt}\left|\mathrm{A}_{1-\delta} \mathrm{X}_{v}\right| \operatorname{Pt}(\beta)$

the relation between the (electronic) thermovoltage $U_{\mathrm{c}}$ and the temperature difference results as

$$
\begin{aligned}
\frac{\mathrm{d} U_{\mathrm{e}}}{\mathrm{d} T} & =\frac{\mathrm{d}\left(\phi^{(\mathrm{\beta})}-\phi^{(\alpha)}\right)}{\mathrm{d} T} \\
& =-\frac{1}{F}\left(\frac{\mathrm{d} \tilde{\mu}_{\mathrm{e}^{-}}^{(\mathrm{Ax})}}{\mathrm{d} T}-\frac{\mathrm{d} \tilde{\mu}_{\mathrm{e}}^{(\mathrm{P})}}{\mathrm{d} T}\right)=\varepsilon_{\mathrm{e}}^{(\mathrm{AX})}-\varepsilon^{(\mathrm{P} 1)}
\end{aligned}
$$

with $\phi$ denoting the Galvani potential in the platinum contacts and with the absolute thermopower defined as $\varepsilon_{\mathrm{e}}^{(\mathrm{AX})} \equiv-1 / F \cdot\left(\mathrm{d} \tilde{\mu}_{\mathrm{e}}^{(\mathrm{AX})} / \mathrm{d} T\right)$ and $\varepsilon^{(\mathrm{Pt})} \equiv$ $-1 / F \cdot\left(\mathrm{d} \tilde{\mu}_{\mathrm{e}^{-}}^{(\mathrm{P}())} / \mathrm{d} T\right)$. Thus, the inert metal is a probe for the electronic properties, exclusively. If cell (I) is in its stationary state $(\nabla T \neq 0)$ under open circuit condition, the fluxes of ions and electrons vanish $\left(j_{\mathrm{A}^{*}}=j_{\mathrm{c}}=0\right)$, and one obtains the following equation for the electronic thermopower from Eq. (4),

$$
\begin{aligned}
\varepsilon_{\mathrm{e}^{-}}^{(\mathrm{AX})}(t \rightarrow \infty) & =-\frac{1}{F} \frac{\mathrm{d} \tilde{\mu}_{\mathrm{e}^{-}}^{(\mathrm{AX})}}{\mathrm{d} T}=\frac{1}{F}\left(\bar{S}_{\mathrm{c}^{-}}+\frac{Q_{\mathrm{c}^{-}}^{*}}{T}\right) \\
& =\frac{1}{F} \cdot S_{\mathrm{e}^{-}}^{*}
\end{aligned}
$$

with $S_{c}^{*}$ defined as the 'entropy of transfer' of electrons. In the very initial state of a thermopower experiment, i.e. if a temperature gradient has just been applied, the crystal is still homogeneous with respect to its composition $(\nabla \delta=0)$. However, thermal diffusion takes place, according to Eq. (7), and the metal excess starts to redistribute by coupled diffusion of ions and electrons. Combining Eqs. (3) and (4) by the coupling condition $j_{\mathrm{A}^{*}}=z \cdot j_{\mathrm{e}}$ and inserting Eq. (6), it follows for the electrochemical potential of the electrons:

$$
\begin{aligned}
\nabla \tilde{\mu}_{\mathrm{c}}= & \frac{t_{\mathrm{A}}}{z}\left(\frac{\partial \mu_{\mathrm{A}}}{\partial \delta}\right)_{T} \nabla \delta \\
& +\left[-\bar{S}_{\mathrm{c}}+\frac{t_{\mathrm{A}}}{z} \cdot \frac{Q_{\mathrm{A}^{-}}^{*}}{T}-t_{\mathrm{c}} \cdot \frac{Q_{\mathrm{c}}^{*}}{T}\right] \nabla T .
\end{aligned}
$$

Assuming a homogeneous composition, $\nabla \delta=0$, one obtains from insertion of Eq. (10) into the definition of the absolute thermopower (see above):

$$
\varepsilon_{\mathrm{c}}^{(\mathrm{AX})}(t \rightarrow 0)=\frac{1}{F}\left(\bar{S}_{\mathrm{e}}-\frac{t_{\mathrm{A}^{*}}}{z} \cdot \frac{Q_{\mathrm{A}^{*+}}^{*}}{T}+t_{\mathrm{c}} \cdot \frac{Q_{\mathrm{e}}^{*}}{T}\right) .
$$

Thus, the thermopower in the initial state of the 
non-isothermal crystal differs from the stationary thermopower in Eq. (9). Subtracting Eqs. (9) and (11), this difference equals

$\varepsilon_{\mathrm{e}^{-}}^{(\mathrm{AX})}(t \rightarrow \infty)-\varepsilon_{\mathrm{e}^{-}}^{(\mathrm{AX})}(t \rightarrow 0)=\frac{1}{z F} \cdot t_{\mathrm{A}^{*}} \frac{Q_{\mathrm{A}}^{*}}{T}$.

An equivalent result can be derived for the case of anion-conducting materials $\mathrm{A}_{1+\delta} \mathrm{X}_{\nu}$ with mobile anions $\mathrm{X}^{z / \nu^{-}}$of the formal valence $z / \nu-$ :

$\varepsilon_{\mathrm{e}^{-}}^{(\mathrm{AX})}(t \rightarrow \infty)-\varepsilon_{\mathrm{e}^{-}}^{(\mathrm{AX})}(t \rightarrow 0)=-\frac{\nu}{z F} \cdot t_{\mathrm{X}=/ \nu} \frac{Q_{\mathrm{X}}^{*}}{T}$.

Applying Eq. (12) or Eq. (13) to materials with a small electronic transference number $\left(t_{\mathrm{A}^{*}} \simeq 1\right.$ or $t_{\mathrm{x}=1}=1$ ), the heat of transport of the mobile component can be extracted directly from a timeresolved measurement of the electronic thermopower. The analysis of such measurement is shown schematically in Fig. 1: The actual temperature difference $\Delta T$ is depicted on the abscissa and the resulting thermovoltage $U_{\mathrm{e}^{-}}$is depicted on the ordinate. To realize the experiment, a temperature gradient has to be applied very quickly, while the thermovoltage is measured continuously. The initial linear increase in region (a), caused by the increasing temperature difference during the establishment of the stationary temperature profile, is determined by Eq. (11), i.e. by the electronic entropy and the ionic

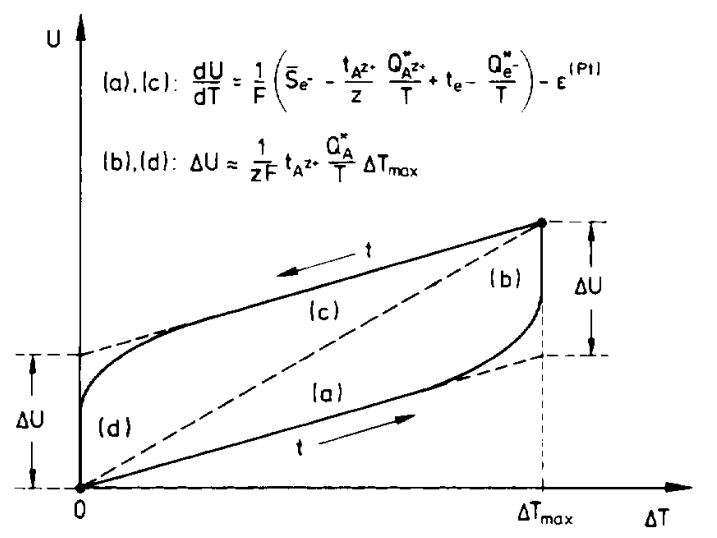

Fig. 1. Time-dependence of the electronic thermopower of a mixed conductor with prevailing ionic conductivity. heat of transport. Since the thermal diffusivity $\lambda / c_{\mathrm{p}}$ ( $\lambda$ : thermal conductivity, $c_{p}$ : specific heat) of most compounds is considerably higher than the chemical diffusion coefficient $\tilde{D}$, the temperature profile becomes usually stationary within a few minutes, and $\Delta T_{\max }$ is observed. In the present case, $\lambda / c_{\mathrm{p}}$ can be estimated as $\lambda / c_{\mathrm{p}} \simeq 0.01 \mathrm{~cm}^{2} \cdot \mathrm{s}^{-1}$ for $\alpha-\mathrm{Ag}_{2+\delta} \mathrm{S}$ and $\alpha-\mathrm{Ag}_{2+\delta} \mathrm{Se}$ and as $\lambda / c_{\mathrm{p}} \simeq 6 \times 10^{-3} \mathrm{~cm}^{2} \cdot \mathrm{s}^{-1}$ for $\mathrm{AgCl}[17,18]$. Thus, $\lambda / c_{\mathrm{p}}$ is comparable to $\tilde{D}_{\mathrm{Ag}}$ in the case of $\alpha-\mathrm{Ag}_{2+\delta} \mathrm{S}$, but a factor of $10^{2}$ higher than $\tilde{D}_{\mathrm{Ag}}$ in $\alpha-\mathrm{Ag}_{2+\delta} \mathrm{Se}$ and $\mathrm{AgCl}$ (cf. also the results).

After the initial linear period, the redistribution of the mobile component leads to a serious deviation of the thermovoltage from the linear behaviour, running into a stationary value at sufficiently long times [region (b)]. As depicted in Fig. 1, the heat of transport $Q_{\mathrm{A}}^{*}$ can be determined directly from the differences $\Delta U_{\mathrm{e}}$ and $\Delta T_{\max }$ by

$\Delta U_{\mathrm{e}}=\frac{1}{z F} \cdot \frac{Q_{\mathrm{A}}^{*}}{T} \cdot \Delta T_{\max } \quad\left(t_{\mathrm{A}^{\ddagger}} \simeq 1\right)$

with $Q_{\mathrm{A}}^{*}$ and $T$ as average values over the respective temperature range. When the additional heating for the establishment of the temperature gradient is removed, the thermovoltage decreases with a constant initial slope [region (c)], which is again given by Eq. (11). The slope is the same as in region (a) of the metal redistribution since, in case of a small applied temperature difference $\left(\Delta T_{\max } / T \ll 1\right)$, the quantities in Eq. (11) do not change significantly. Finally, the material homogenizes [region (d)] and a zero EMF is again observed. In region (d), the same difference $\Delta U_{\mathrm{t}}$ as found in region (b) is observed. The behaviour of the thermovoltage with time is indicated by arrows in Fig. 1. Since the redistribution starts immediately after the application of the temperature gradient, periods (a) and (c) are only very short in time. Thus, the rate of taking data points has to be high enough in order to detect the linear regimes. Essentially, the ratio of the thermal conductivity and the chemical diffusion coefficient decides whether the experiment is possible or not. Since the thermal conductivities of ionic crystals do not differ too much from each other, the magnitude of the chemical diffusion coefficient is critical. 


\subsection{Measurements with ion-conducting electrodes}

For a thermogalvanic cell involving a mixed conducting compound $\mathrm{A}_{1+\delta} \mathrm{X}_{v}$ with ion-conducting electrodes (AY: solid electrolyte) of the type

$(\alpha) \operatorname{Pt}|\mathrm{A}| \mathrm{AY}\left|\mathrm{A}_{1+\delta} \mathrm{X}_{\nu}\right| \mathrm{AY}|\mathrm{A}| \mathrm{Pt}(\beta)$

the relation between the thermovoltage $U_{\mathrm{A}^{i}+}$ and the temperature difference results as

$$
\begin{aligned}
\frac{\mathrm{d} U_{\mathrm{A}^{*+}}}{\mathrm{d} T} & =\frac{\mathrm{d}\left(\phi^{(\beta)}-\phi^{(\alpha)}\right)}{\mathrm{d} T}=\varepsilon_{\mathrm{A}^{*+}}^{(\mathrm{Ax})}-\varepsilon^{\left(\mathrm{P}^{+}\right)} \\
& =\frac{1}{F}\left(\frac{\mathrm{d} \tilde{\mu}_{\mathrm{A}^{*}}^{(\mathrm{AX})}}{\mathrm{d} T}+S_{\mathrm{A}}^{0}+\frac{\mathrm{d} \tilde{\mu}_{\mathrm{c}^{-}}^{(\mathrm{Pt})}}{\mathrm{d} T}\right),
\end{aligned}
$$

if one assumes that the ionic electrodes themselves are not exposed to any temperature gradient $\left(S_{\mathrm{A}}^{0}\right.$ : entropy of pure metal A). In formal accord with the definition of the electronic thermopower, the ionic thermopower shall be defined by Eq. (16) as

$\varepsilon_{\mathrm{A}^{*}+}^{(\mathrm{AXX})} \equiv \frac{1}{F}\left(\frac{\mathrm{d} \tilde{\mu}_{\mathrm{A}^{*}+}^{(\mathrm{AX})}}{\mathrm{d} T}+S_{\mathrm{A}}^{0}\right)$,

i.e. the electron-blocking electrodes are probes for the ionic properties. The inclusion of the metal entropy $S_{\mathrm{A}}^{0}$ into the definition of $\varepsilon_{\mathrm{A}^{*}+}^{(\mathrm{AX})}$ is arbitrary, but simplifies the formalism. Under open circuit conditions and in the stationary state, $j_{\mathrm{A} z+}=z$. $j_{\mathrm{e}^{-}}=0$, the ionic thermovoltage follows from Eqs.

(3) and (16) as

$$
\begin{aligned}
\varepsilon_{\mathrm{A}^{i-}}^{(\mathrm{AX})}(t \rightarrow \infty) & =-\frac{1}{z F}\left(\bar{S}_{\mathrm{A}^{i+}}-S_{\mathrm{A}}^{0}+\frac{Q_{\mathrm{A}^{*+}}^{*}}{T}\right) \\
& =-\frac{1}{z F}\left(S_{\mathrm{A}^{i+}}^{*}-S_{\mathrm{A}}^{0}\right),
\end{aligned}
$$

in formal accord with Eq. (9), and with $S_{A^{*}+\text { defined }}^{*}$ as the 'entropy of transfer' of ions. In the initial state during the establishment of the temperature gradient $(\nabla \delta=0)$, the metal excess starts to redistribute by coupled fluxes $\left(j_{\mathrm{A}^{*+}}=z \cdot j_{\mathrm{e}^{-}}\right)$, and it follows for the electrochemical potential of the ions [combining Eq. (3), Eq. (4) and Eq. (6)]:

$$
\begin{aligned}
\nabla \tilde{\mu}_{\mathrm{A}^{*+}}= & t_{\mathrm{c}^{-}} \cdot\left(\frac{\partial \mu_{\mathrm{A}}}{\partial \delta}\right)_{T} \nabla \delta \\
& +\left[t_{\mathrm{e}} \cdot \frac{Q_{\mathrm{A}}^{*}}{T}-\left(\bar{S}_{\mathrm{A}^{z^{+}}}+\frac{Q_{\mathrm{A}^{*+}}^{*}}{T}\right)\right] \nabla T .
\end{aligned}
$$

Assuming again that the chemical potential gradient is only given by the partial entropy term in Eq. (6), i.e. setting $\nabla \delta=0$, one obtains from insertion of Eq. (18) into Eq. (16)

$$
\begin{aligned}
\varepsilon_{\mathrm{A}^{*+}}^{(\mathrm{Ax})}(t \rightarrow 0)= & -\frac{1}{z F}\left(\bar{S}_{\mathrm{A}^{*+}}-S_{\mathrm{A}}^{0}+t_{\mathrm{A}^{*}+} \cdot \frac{Q_{\mathrm{A}^{*+}}^{*}}{T}\right. \\
& \left.-z \cdot t_{\mathrm{e}^{-}} \cdot \frac{Q_{\mathrm{c}^{-}}^{*}}{T}\right) .
\end{aligned}
$$

Subtracting Eqs. (19) and (17), the difference between the initial ionic thermopower and the thermopower in the stationary state equals

$\varepsilon_{\mathrm{A}^{*}+}^{(\mathrm{AX})}(t \rightarrow \infty)-\varepsilon_{\mathrm{A}^{*}}^{(\mathrm{AX})}(t \rightarrow 0)=\frac{1}{z F} \cdot t_{\mathrm{e}^{-}} \frac{Q_{\mathrm{A}}^{*}}{T}$.

For an anion-conducting material $\mathrm{A}_{1+\delta} \mathrm{X}_{\nu}$ with mobile anions $\mathrm{X}^{\nu-}$ of the formal valence $\nu$, the equivalent expression results as:

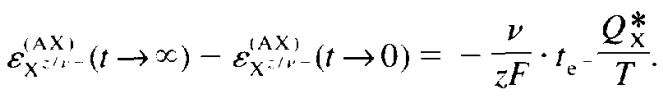

Applying Eq. (20) or Eq. (21) to materials with small ionic transference number $\left(t_{\mathrm{c}^{-}} \simeq 1\right)$, the heat of transport of a mobile component can be extracted from a time-resolved thermopower measurement with ionic electrodes, without the need for additional data. The analysis of the time-dependent ionic thermovoltage is equivalent to the one given above for the electronic thermopower.

In Figs. 2 and 3, the possible results from simultaneous measurements of ionic and electronic thermovoltages are depicted for different materials. Thus, Fig. 2 comprises the case of an electronic conductor with small ionic conductivity, and Fig. 3 shows the case of an ionic conductor with small electronic conductivity. Depending on the magnitude and the sign of the heat of transport, different characteristic 'thermopower hysteresises' can be observed. Combining the different thermovoltages, as indicated in Figs. 2 and 3, the heat of transport, the partial entropy and the entropy of transfer can be obtained.

If the material of interest is a mixed conductor with comparable ionic and electronic conductivity $\left(t_{\mathrm{A}^{*}} \simeq t_{\mathrm{e}}\right)$, the analysis of the thermopower hyster- 


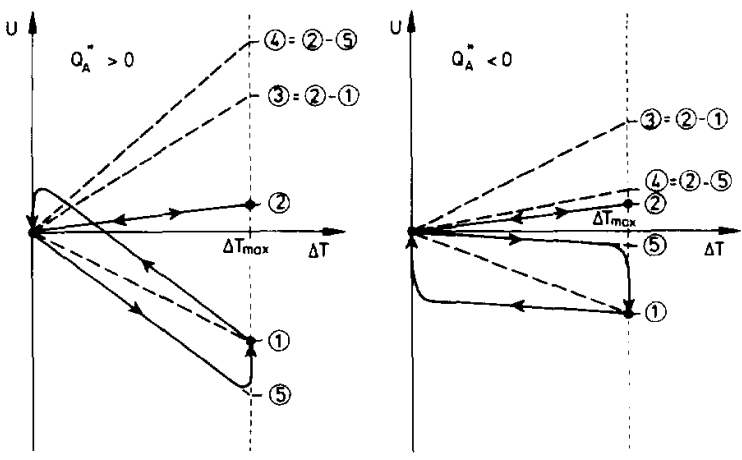

Fig. 2. Evaluation of the heat of transport from time-dependent measurements of the partial ionic and electronic thermopower for a semiconductor with $t_{\mathrm{A}^{*}} \ll t_{\mathrm{e}}:$ : (a) $Q_{\mathrm{A}}^{*}>0$, (b) $Q_{\mathrm{A}}^{*}<0$. The numbers denote the following quantities: (1) $\varepsilon_{\mathrm{A}^{*}}(t \rightarrow \infty)=$ $-(1 / z F)\left(S_{\mathrm{A}^{-+}}^{*}-S_{\mathrm{A}}^{13}\right),(2) \varepsilon_{\mathrm{c}^{-}}=(1 / F) S_{\mathrm{i}^{-}}^{*},(3)(\mathrm{d} U / \mathrm{d} T)_{\mathrm{A}^{\mathrm{A}}=0}=(1 /$ $z F)\left(S_{\mathrm{A}}^{*}-S_{\mathrm{A}}^{11}\right),(4)(\partial U / \partial T)_{\delta}=(1 / z F)\left(S_{\mathrm{A}}-S_{\mathrm{A}}^{\prime \prime}\right),(5) \varepsilon_{\mathrm{A}}-(t \rightarrow 0)=$ $(1 / F) S_{E^{-}}^{*}-(1 / z F)\left(S_{A}-S_{A}^{0}\right)$.

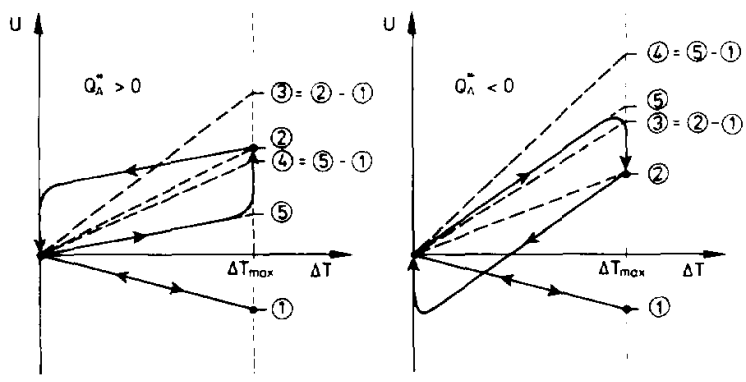

Fig. 3. Evaluation of the heat of transport from time-dependent measurements of the partial ionic and electronic thermopower for an ionic conductor with $t_{\mathrm{e}^{-}} \ll t_{\mathrm{A}^{+}}$: (a) $Q_{\mathrm{A}}^{*}>0$, (b) $Q_{\mathrm{A}}^{*}<0$. The numbers denote the following quantities: (1) $\varepsilon_{\mathrm{A}^{*}}=-(1 /$ $z F)\left(S_{\mathrm{A}^{*}-}^{*}-S_{\mathrm{A}}^{1)}\right)$, (2) $\varepsilon_{\mathrm{c}}-(t \rightarrow \infty)=(1 / F) S_{\mathrm{e}}^{*},(3)(\mathrm{d} U / \mathrm{d} T)_{\mathrm{A}_{\mathrm{A}}=0}=$ $(1 / z F)\left(S_{\mathrm{A}}^{*}-S_{\mathrm{A}}^{0}\right), \quad(4) \quad(\partial U / \partial T)_{\dot{\delta}}=(1 / z F)\left(\bar{S}_{\mathrm{A}}-S_{\mathrm{A}}^{(1)}\right), \quad$ (5) $\varepsilon_{\mathrm{c}} \cdot(t \rightarrow 0)=-(1 / z F)\left(S_{\mathrm{A}^{*}}^{*}-S_{\mathrm{A}}^{0}\right)+(1 / z F)\left(\bar{S}_{\mathrm{A}}-S_{\mathrm{A}}^{0}\right)$.

esis becomes less straightforward, and additional information on the transference number of either electrons or ions is necessary for the evaluation of $Q_{A}^{*}$ from a single thermopower measurement, cf. Eqs. (12) and (20) or Eqs. (13) and (21). Alternatively, the chemical potential difference can be measured time-resolved by chemical potential probes, see Fig. 2, i.e. by combining both the ionic and the electronic thermovoltage. Again, this procedure works only for materials with chemical diffusion coefficients that are not too high. If the chemical diffusion coefficient is too high compared to the thermal diffusivity, one has to measure the chemical potential difference in the stationary state and has to determine additional data for the partial entropy of the mobile component. A very elegant experimental solution to the measurement of $Q_{\mathrm{Ag}}^{*}$ in $\beta-\mathrm{Ag}_{2+\delta} \mathrm{S}$, which shows a very high chemical diffusion coefficient, has been realized by Miyatani [4]: He combined the thermal diffusion experiment with a simultaneous entropy measurement on two small sulfide crystals which were separated from the nonisothermal crystal, but always at the same temperature as the ends of the non-isothermal crystal.

A final comment has to be given on the relation between the different measurable quantities: As visualized in Fig. 4 and already indicated in Figs. 2 and 3 , the experimental quantities measureable by electrochemical probes are not independent. Combining Eqs. (9) and (17), one finds that the subtraction of the ionic and electronic thermopower in the initial state $(t \rightarrow 0)$ equals the partial entropy of the mobile component (reduced by the entropy of pure metal A):

$$
\begin{aligned}
& \varepsilon_{\mathrm{A}^{*}}^{(\mathrm{AX})}(t \rightarrow 0)-\varepsilon_{\mathrm{c}^{-}}^{(\mathrm{AX})}(t \rightarrow 0) \\
& \quad=\frac{1}{z F}\left[\left(\frac{\partial \mu_{\mathrm{A}}}{\partial T}\right)_{\delta}+S_{\mathrm{A}}^{0}\right]=-\frac{1}{z F}\left(\bar{S}_{\mathrm{A}}-S_{\mathrm{A}}^{0}\right) .
\end{aligned}
$$

Combining Eqs. (11) and (19), one finds that the subtraction of the ionic and electronic thermopower in the stationary state $(t \rightarrow 0)$ equals the sum of partial entropy and heat of transport of the mobile component (reduced by the entropy of pure metal):

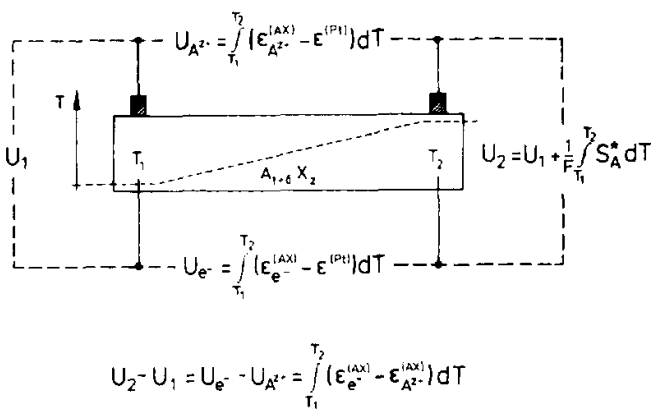

Fig. 4. Schematic arrangement for the measurement of ionic and electronic thermovoltages, or the chemical potential gradient, respectively. 


$$
\begin{aligned}
& \varepsilon_{\mathrm{A}^{*}-}^{(\mathrm{AX})}(t \rightarrow \infty)-\varepsilon_{\mathrm{e}^{-}}^{(\mathrm{AX})}(t \rightarrow \infty) \\
& \quad=\frac{1}{z F}\left[\left(\frac{\mathrm{d} \mu_{\mathrm{A}}}{\mathrm{d} T}\right)_{j_{\mathrm{A}}=0}+S_{\mathrm{A}}^{0}\right] \\
& =-\frac{1}{z F}\left(\bar{S}_{\mathrm{A}}-S_{\mathrm{A}}^{0}+\frac{Q_{\mathrm{A}}^{*}}{T}\right) \\
& =-\frac{1}{z F}\left(S_{\mathrm{A}}^{*}-S_{\mathrm{A}}^{0}\right) .
\end{aligned}
$$

Essentially, the determination of the heat of transport by the partial thermopowers is based on the fact that, depending on the predominant type of conductivity, a gradient of the chemical potential $\mu_{\mathrm{A}}$ of the mobile component in a binary system $\mathrm{A}_{1+\delta} \mathrm{X}_{\nu}$ can be approximated by the gradient of the electrochemical potential of the less mobile charge carrier. Strictly, the relations

$\nabla \tilde{\mu}_{\mathrm{e}^{-}}=\frac{t_{\mathrm{A}^{:-}}}{z} \cdot \nabla \mu_{\mathrm{A}^{\prime}}, \quad \nabla \tilde{\mu}_{\mathrm{A}^{:-}}=t_{\mathrm{e}^{-}} \cdot \nabla \mu_{\mathrm{A}}$

hold. For materials with predominant electronic conductivity the chemical potential gradient can be measured by the corresponding ionic potential gradient,

$\nabla \tilde{\mu}_{\mathrm{A}^{i}} \approx \nabla \mu_{\mathrm{A}} \quad\left(t_{\mathrm{e}^{-}} \gg t_{\mathrm{A}^{+}}\right)$, whereas for materials with predominant ionic conductivity we can assume the relation

$\nabla \tilde{\mu}_{\mathrm{e}^{-}} \simeq \frac{\mathrm{I}}{z} \nabla \mu_{\mathrm{A}} \quad\left(t_{\mathrm{A}}^{z^{+}} \gg t_{\mathrm{e}}\right)$.

In Table 1, the different formal results are summarized.

\section{Experimental}

Thermopower measurements were performed in different furnaces of which one was specially designed for experiments in temperature gradients (different temperature zones) and the other was equipped with an additional small resistance heater at one end of the crystalline specimen. The electric power for these small heaters was supplied by batteries. In the case of $\alpha-\mathrm{Ag}_{2+\delta} \mathrm{Se}$, the construction included a cooling element at the other side of the crystal (silicone oil as cooling liquid), to improve the build-up of the temperature gradient. In general, the establishment of a stationary temperature gradient took from several minutes up to half an hour,

Table 1

Relations for the evaluation of thermopower measurements with two types of electrodes for materials with different conductivity behaviour

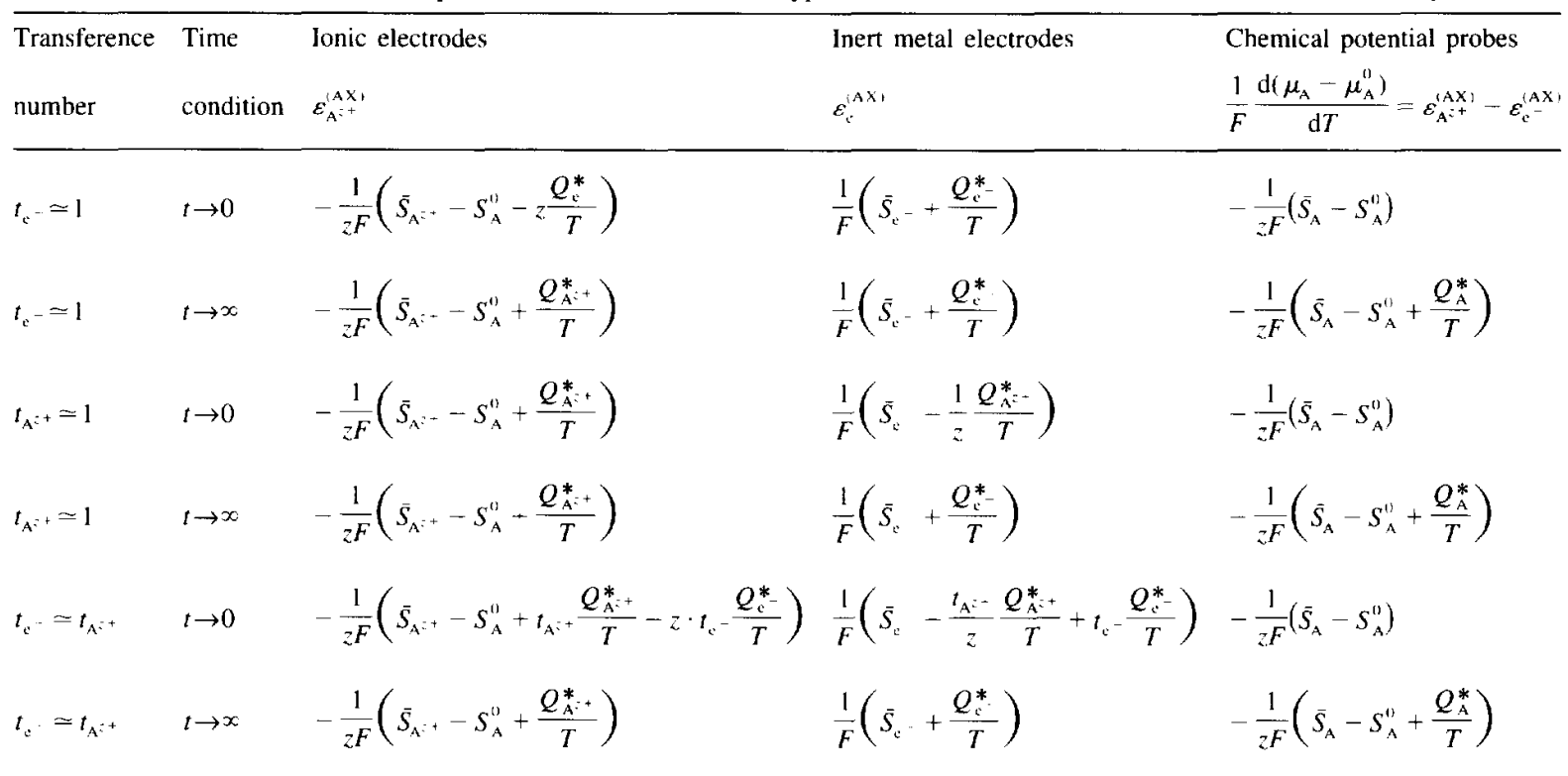


depending on the crystal length. Details of the experimental arrangements can be found in $[2,3]$.

In the case of $\mathrm{AgCl}$, platinum foils were used as electrodes. Thermocouples were attached directly to these electrodes. The thermovoltage was measured with a voltmeter with high input impedance.

In the case of $\alpha-\mathrm{Ag}_{2+\delta} \mathrm{S}$ and $\alpha-\mathrm{Ag}_{2+\delta} \mathrm{Se}$, the electronic thermovoltage was measured by small platinum wires which were grown into the crystals during the crystal growth from the elements. The ionic thermovoltage was measured by small ionic probes (diameter $1 \mathrm{~mm}$ ) of the type $\mathrm{Pt}|\mathrm{Ag}| \mathrm{AgI}$ or $\mathrm{Pt}|\mathrm{Ag}| \mathrm{RbAg}_{4} \mathrm{I}_{5}$, which were placed at the same position as the platinum probes. The temperature was determined by thermocouples which were in direct contact with the chalcogenide crystals at the positions of the potential probes. Additional coulometric titration cells served for the control of the crystal composition. The whole cell was encapsulated in a gas tight cover (glass, PTFE and silicone rubber), to prevent transport via the gas phase.

\section{1. $\alpha-A g_{3+\delta} S$}

The low-temperature phase of silver sulfide is a Boltzmann-type semiconductor with an extremely narrow phase width [19]. A pronounced Frenkel disorder in the cation sublattice leads to a high ionic conductivity. Both the high electronic and ionic conductivity and the large thermodynamic factor lead to a very high, but activity dependent, chemical diffusion coefficient in the order of $\tilde{D}_{\mathrm{Ag}} \simeq 0.1 \mathrm{~cm}^{2}$. $\mathrm{s}^{-1}$ (e.g., $\tilde{D}_{\mathrm{Ag}}=0.16 \mathrm{~cm}^{2} \cdot \mathrm{s}^{-1}$ at $T=168^{\circ} \mathrm{C}$ and $\delta \simeq 0$ [20]). Thus, even in crystals several centimeters in length, stationary states are generally attained with a few minutes. The crystal was $2.2 \times 2.6 \times 14 \mathrm{~mm}^{3}$ in size and grown from the elements at $170^{\circ} \mathrm{C}$. Thus, it has never been transformed to the stucturally disordered $\beta$-phase. The probe distance was $12 \mathrm{~mm}$.

$$
\text { 3.2. } \alpha-A g_{2+\delta} S e
$$

The low-temperature phase of silver selenide is a semicondu. " th a phase width in the order of $-1 \times 10^{-4}<\delta<6.5 \times 10^{-4} \quad\left(T=100^{\circ} \mathrm{C} ;[21,22]\right.$. A pronounced Frenkel disorder in the cation sublattice leads to a high ionic conductivity. For the electronic transference number we can, however, assume a value of $t_{\mathrm{e}^{-}} \simeq 1$. The chemical diffusion coefficient is in the order of $\tilde{D}_{\mathrm{Ag}} \simeq 10^{-4} \mathrm{~cm}^{2} \cdot \mathrm{s}^{-1}$ (e.g., $\tilde{D}_{\mathrm{Ag}} \simeq$ $1.4 \times 10^{-4} \mathrm{~cm}^{2} \cdot \mathrm{s}^{-1}$ at $T=106^{\circ} \mathrm{C}$ and $\delta=3 \times 10^{-5}$ [23]), and thus, is considerably smaller than the chemical diffusion coefficient of $\alpha-\mathrm{Ag}_{2}+\delta$ S. The $\alpha-$ $\mathrm{Ag}_{2+\delta} \mathrm{S}$ crystal was $2.2 \times 2.2 \times 9 \mathrm{~mm}^{3}$ in size and grown from the elements at $400^{\circ} \mathrm{C}$. Stationary states were generally attained within $12 \mathrm{~h}$. The probe distance was $7 \mathrm{~mm}$.

\section{3. $\mathrm{AgCl}$}

Silver chloride shows silver ion conductivity due to the Frenkel disorder in the cation sublattice [24]. The electronic conductivity is very small, leading to an electronic transference number in the order of $t_{\mathrm{c}^{-}} \simeq 10^{-5}[24,25]$. The deviation from stoichiometry is extremely small and not reliably known. Data for the chemical diffusion coefficient are not reported in the literature. We estimate a thermodynamic factor in the order of $\left(\partial \mu_{\mathrm{Ag}} / \partial \delta\right)_{T} \simeq 10^{10} \mathrm{~kJ} \cdot \mathrm{kmol}^{-1}$, which leads, together with data for the electronic conductivity [25], to a value of $\tilde{D}_{\mathrm{Ag}}$ in the order of $10^{-4}$ $\mathrm{cm}^{2} \cdot \mathrm{s}^{-1}$. This compares reasonably with the value of $\tilde{D}_{\mathrm{Ag}} \simeq 2 \times 10^{-4} \mathrm{~cm}^{2} \cdot \mathrm{s}^{-1}$, which can be estimated from the time needed for the attainment of the stationary state in our thermal diffusion experiments on $\mathrm{AgCl}$.

\section{Results and discussion}

In Figs. 5-8, typical results from the measurements of ionic and electronic thermovoltages are

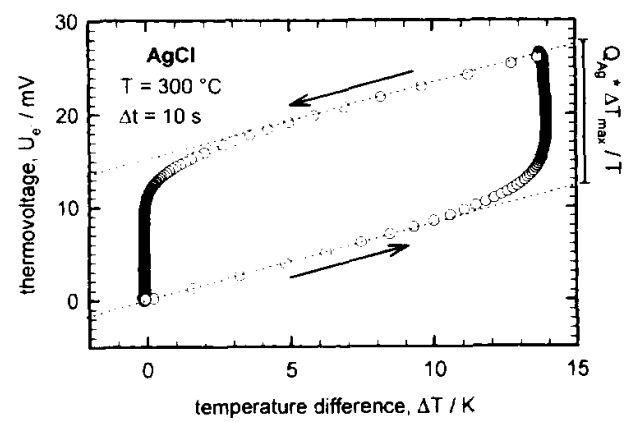

Fig. 5. Measurement of the electronic thermopower of single crystalline $\mathrm{AgCl}$ 


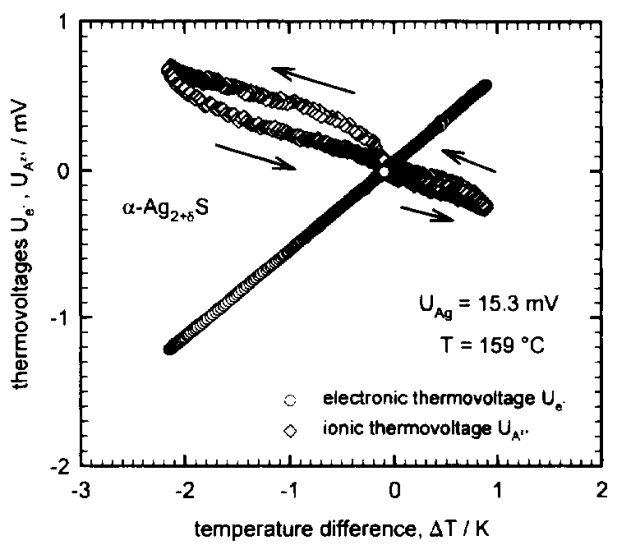

Fig. 6. Measurement of the ionic and electronic thermopower of $\alpha-\mathrm{Ag}_{2+\delta} \mathrm{S}$; experimental conditions are denoted in the figure.

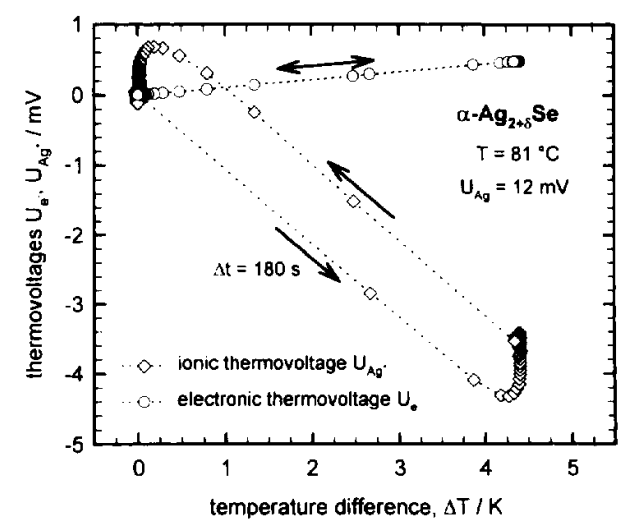

Fig. 7. Measurement of the ionic and electronic thermopower of $\alpha-\mathrm{A}_{2+8} \mathrm{Se}$; experimental conditions are denoted in the figure.

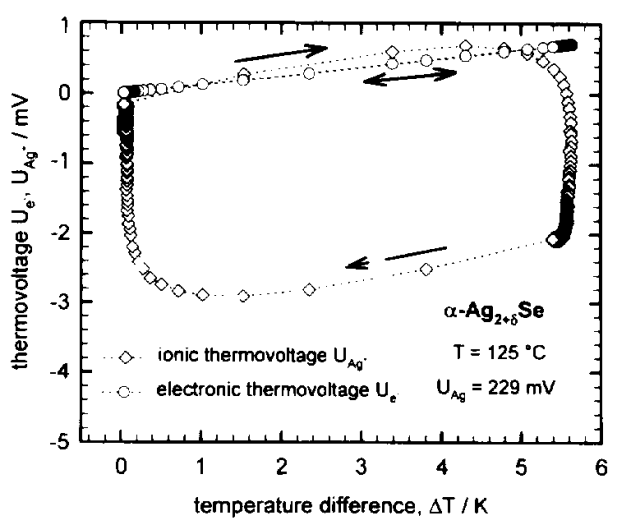

Fig. 8. Measurement of the ionic and electronic thermopower of $\alpha-\mathrm{Ag}_{2+\delta} \mathrm{Se}$; experimental conditions are denoted in the figure. depicted for the three different materials. The results prove that the time-dependent measurement of partial thermovoltages on crystals which are closed for matter exchange, provides a suitable method for the quantitative measurement of heats of transport. Particularly, the electronic thermovoltage curves ('thermopower hysteresis') for $\mathrm{AgCl}$ (Fig. 5) and the ionic thermovoltage curves for $\alpha-\mathrm{Ag}_{2+\delta} \mathrm{Se}$ (Figs. 7 and 8) agree with the predicted behaviour in Figs. 1-3, i.e. the redistribution kinetics is sufficiently slow compared to the establishment of stationary thermal states, and the heats of transport can be evaluated. The measurement failed for $\alpha-A_{2+\delta} S$, i.e. the thermopower hysteresis could not be evaluated, since the temperature gradient could not be established fast enough. In fact, $\alpha-\mathrm{Ag}_{2+\delta} \mathrm{S}$ exhibits an exceptionally high diffusion coefficient, and experimental determinations of $Q_{\mathrm{Ag}}^{*}$ have to rely on the study of the stationary state. Most other systems of interest will show much smaller diffusion coefficients.

Another general problem of the proposed measurements is related to the charge transfer equilibrium at the electrodes: It is favorable for the quantitative evaluation of $Q_{\mathrm{A}}^{*}$ if either the ionic or the electronic transference number is nearly one. This may cause problems in the EMF measurement in extreme cases, since a minimum charge carrier concentration is necessary to establish electrochemical equilibrium at the electrodes. In this respect, the measurement of electronic thermovoltages in $\mathrm{AgCl}$ represents, already, a critical case. The electronic transference number in $\mathrm{AgCl}$ under the experimental conditions is in the order of $t_{\mathrm{e}^{-}} \simeq 10^{-5}[24,25]$ and thus, the cell impedance was very high, leading to EMF drifts even without an applied temperature gradient. Careful electromagnetic shielding can reduce this problem, but will not remove these disturbances completely.

A more technical problem concerns the establishment of temperature gradients. Only if the thermogalvanic cell, its electric contacts and the surrounding heating elements are carefully designed, the establishment of a temperature profile occurs uniformly. This is not critical in the study of stationary states but becomes important in the study of transient phenomena.

Finally, the validity of the experimental results depends strongly on the boundary condition of 
crystal surfaces that are closed for matter exchange. In the present case of experiments at relatively low temperatures, the cover of the crystals by inert materials causes no problem, and the electrode materials ( $\mathrm{Pt}, \mathrm{AgI}, \mathrm{Ag}_{4} \mathrm{RbI}_{5}$ ) are also impermeable for the components. However, experiments on other systems at higher temperatures and particularly on oxides, will need a careful design to realize comparable boundary conditions. To provide an example, the solubility of many transition metals in platinum is so large (increasing especially at lower oxygen activities) that platinum can probably not be used as an inert cover or electrode material for Soret experiments on the corresponding oxides (e.g., iron and manganese oxides).

Data for the heats of transport $Q_{\mathrm{Ag}}^{*}$ of silver metal have not been reported to date for all three investigated materials. However, studies of the ionic thermopower in pure and doped $\mathrm{AgCl}$ have been performed by various authors, leading to information on the sum of the heats of transport $Q_{\mathrm{v}}^{*}+Q_{\mathrm{i}}^{*}$ of vacancies and interstitials (e.g., [29,30]). The analysis of our measurement in Fig. 4 by the use of Eq. (12) leads to a value of $Q_{\mathrm{Ag}}^{*}=62.1 \mathrm{~kJ} \cdot \mathrm{mol}^{-1}$ for $\mathrm{AgCl}\left(t_{\mathrm{A}^{-}} \approx 1\right)$. If we assume that the electronic contribution is only in the order $R T$, we can approximate $Q_{\mathrm{Ag}^{+}}^{*} \simeq Q_{\mathrm{Ag}}^{*}$. The validity of this assumption depends on the nature of the electronic charge carriers, thus it can be used safely only for delocalised (non-polaronic) charge carriers. Since the silver chloride crystal was equilibrated with silver metal before the experiment, the electronic defects are electrons and the crystal is a n-type semiconductor (cf. [25]). According to Haynes and Shockley [26], the mobility of electronic defects in $\mathrm{AgCl}$ equals $u_{\mathrm{n}}=35 \mathrm{~cm}^{2} \cdot(\mathrm{V} \cdot \mathrm{s})^{-1}$ at $T=100^{\circ} \mathrm{C}$, with the tendency to decrease with increasing temperature. Thus, by extrapolation of the data in [26] to $T=300^{\circ} \mathrm{C}$ we estimate a value of $u_{\mathrm{n}}=20 \mathrm{~cm}^{2} \cdot(\mathrm{V} \cdot \mathrm{s})^{-1}$, which indicates a non-polaronic character of the electrons. Haynes and Shockley draw the same conclusion and even further conclude that the scattering of the electrons in $\mathrm{AgCl}$ is caused by optical phonons of the lattice. The corresponding scattering factor in the statistical approach to the electronic thermopower equals $r_{\mathrm{n}}=-\frac{1}{2}$ [27], which leads to a heat of transport of $Q_{\mathrm{n}}^{*}=-\frac{1}{2} R T$, thus validates our assumption that $Q_{\mathrm{Ag}^{-}}^{*} \cong Q_{\mathrm{Ag}}^{*}$ (see Ref. [2] for a discussion of electronic heats of transport).
According to previous analyses of ionic heats of transport in crystals with Frenkel disorder and intrinsic defect concentrations $[15,16] Q_{\mathrm{Ag}^{+}}^{*}$ is composed as

$Q_{\mathrm{Ag}}^{*}=\frac{\psi_{\mathrm{i}}}{\psi_{\mathrm{i}}+1} Q_{\mathrm{i}}^{*}-\frac{1}{\psi_{\mathrm{i}}+1} Q_{\mathrm{v}}^{*}+\frac{\psi_{\mathrm{i}}-1}{\psi_{\mathrm{i}}+1} \cdot \frac{\Delta H_{\mathrm{F}}}{2}$.

Inserting the values for the Frenkel enthalpy $\Delta H_{\mathrm{F}}=1.61 \mathrm{eV}$ and the mobility ratio $\psi_{\mathrm{i}} \equiv u_{\mathrm{i}} / u_{\mathrm{v}}=$ 5.96 of interstitials and vacancies [28], we obtain the relation

$0.856 \cdot Q_{\mathrm{i}}^{*}-0.144 \cdot Q_{\mathrm{v}}^{*} \simeq 6.75 \mathrm{~kJ} \cdot \mathrm{mol}^{-1}$.

Christy reports the sum $Q_{\mathrm{i}}^{*}+Q_{\mathrm{v}}^{*}=-82 \mathrm{~kJ} \cdot \mathrm{mol}^{-1}$ for $\mathrm{AgCl}$, obtained from measurements of the ionic thermopower of doped and undoped $\mathrm{AgCl}$. Combining Christy's and our result, we evaluate the following data for the heats of transport of interstitials and vacancies in $\mathrm{AgCl}$ at $300^{\circ} \mathrm{C}$ :

$Q_{\mathrm{i}}^{*} \simeq-5 \mathrm{~kJ} \cdot \mathrm{mol}^{-1}$,

$Q_{\mathrm{v}}^{*} \simeq-77 \mathrm{~kJ} \cdot \mathrm{mol}^{-1}$.

It has to be emphasized that the experimental information on the heats of transport of interstitials and vacancies from Christy's and our experimental results is independent. However, the evaluation is not based on pure experimental grounds, rather it implies an estimation of the electronic heat of transport.

In the case of $\alpha-\mathrm{Ag}_{2+\delta} \mathrm{Se}$, see Fig. 7, we obtain a value of $Q_{\mathrm{Ag}}^{*}=-12.8 \mathrm{~kJ} \cdot \mathrm{mol}^{-1}$ for the heat of transport at an EMF of $12 \mathrm{mV}$ and at $T=82^{\circ} \mathrm{C}$ by the use of Eq. (20). A value of $Q_{\mathrm{Ag}}^{*}=22.7 \mathrm{~kJ} \cdot \mathrm{mol}^{-1}$ at an EMF of $229 \mathrm{mV}$ and at $T=125^{\circ} \mathrm{C}$ can be evaluated from the results in Fig. 8. As shown in a forthcoming study on the non-isothermal transport properties of $\alpha-\mathrm{Ag}_{2+\delta} \mathrm{Se}$, this strong dependence of the heat of transport on the thermodynamic variables (particularily on the composition) can be understood completely on the basis of the defect structure. Assuming again a negligible electronic contribution, we can identify the measured heat of transport with the ionic heat of transport, thus $Q_{\mathrm{Ag}^{+}}^{*} \simeq Q_{\mathrm{Ag}}^{*}$. In contrast to $\mathrm{AgCl}$, the ionic defect concentrations in $\alpha-\mathrm{Ag}_{2+\delta}$ Se vary as a function of the silver activity, and Eq. (27) can only be used at the exact stoichio- 
metric composition. However, at low EMF values, such as $12 \mathrm{mV}$ in Fig. 7, the interstitial conductivity is predominant, and in this case the ionic heat of transport is determined by the interstitial heat of transport:

$Q_{\mathrm{i}}^{*} \simeq Q_{\mathrm{Ag}^{+}}^{*} \simeq Q_{\mathrm{Ag}}^{*}=-12.8 \mathrm{~kJ} \cdot \mathrm{mol}^{-1}$.

The analysis of the result in Fig. 8 in terms of defect properties is less straightforward, since at high EMF values both interstitials and vacancies are present. Explicitly, data for the Frenkel enthalpy, the mobility ratio and the concentration ratio of interstitials and vacancies are necessary for a quantitative interpretation [3].

In respect to our measurements of the electronic thermopower on the ionic conductor $\mathrm{AgCl}$, experiments by Yoo and co-workers on the superionic conductor $\alpha$-AgI have to be mentioned. Since thermopower measurements on AgI with inert metal electrodes failed, due to the extremely small concentration of electronic charge carriers [31], Yoo and co-workers used silver sulfide as a mixed conducting electrode [9]. Combining different thermopower measurements, they were then able to evaluate information on the heat of transport of silver metal in AgI.

Besides this study, only a minor number of experiments have been performed which apply the time-dependent measurement of thermovoltages to the determination of the heat of transport. In none of these studies has the boundary condition of closed surfaces, which is necessary for a well defined Soret experiment, been strictly realized. For example, Yoo and Hwang [13] study the change of the electronic thermopower in yttria-doped zirconia after a heat pulse at one side of the crystal. Since the outer surfaces of the zirconia crystal were exposed to the gas phase, the entropy of gaseous oxygen determines the redistribution of the oxygen component in the crystal and Eq. (13) does not apply. However, using an appropriate formal analysis, i.e. taking the equilibrium with the gas phase into account, information on $Q_{A}^{*}$ has been obtained. Hodge and Bowen [33] studied the electronic thermovoltage of $\mathrm{Fe}_{1-x} \mathrm{O}$ under comparable conditions, but did not perform a quantitative analysis. Millot studied the redistribution of oxygen in $\mathrm{CeO}_{2-x}$ and $(\mathrm{Ce}, \mathrm{U})_{2-x}$ by the measurement of the ionic thermovoltage with zirconia electrodes and determined data for the heat of transport $Q_{\mathrm{O}}^{*}$ of oxygen $[11,12]$.

Summing up, it may be said that the measurement of time-dependent thermovoltages provides a suitable means for the study of thermal diffusion. If one is only interested in precise thermopower measurements rather than in thermal diffusion, the change of either the electronic or the ionic thermovoltage due to the redistribution of a mobile component should always be taken into account as a possible source of errors. As Eqs. (12) and (20) show, this will not be a problem in the study of the electronic thermopower of semiconducting compounds with very small ionic transference numbers (e.g., transition metal oxides $\mathrm{FeO}, \mathrm{CoO}, \mathrm{MnO}$, etc.), nor in the study of the ionic thermopower of solid electrolytes $\left(t_{\mathrm{A}^{*}} \simeq 1\right)$ with reversible metal electrodes (e.g., silver electrodes for silver halides). Rather one has to be careful in the study of real mixed conducting compounds, i.e. of materials with comparable ionic and electronic conductivity. Here the Soret effect may cause serious disturbances in the same order of magnitude as the thermovoltage itself.

In [14], Wagner is sceptical about the validity of time-dependent thermopower measurements, and therefore, gives no complete analysis of the thermopower at short times. His scepticism centers on the fact that a crystal which is exposed to a temperature gradient suffers from mechanical stress due to varying thermal expansion along the gradient. Definitely, this initial mechanical stress will cause an additional effect on the component redistribution. And since the elastic stress can relax during the attainment of the stationary state by diffusional creep or by dislocation motion, one may argue that, in particular, transient measurements lead to an error in the evaluation of the heat of transport. However, our present results lead us to the conclusion that elastic effects will not cause serious errors in the determination of the heat of transport: Comparing the results from time-dependent measurements and from the analysis of stationary states in the case of $\alpha-\mathrm{Ag}_{2+\delta} \mathrm{Se}$, we observe no difference in the heats of transport.

\section{Conclusions}

Time-dependent measurements of partial thermovoltages provide a direct route for the experimen- 
tal determination of heats of transport of mobile components in mixed conductors in a single experiment. The experimental analysis is straightforward for materials with either small ionic or electronic transference number. As an important boundary condition, the crystal has to be closed for matter exchange at all its surfaces. For materials with comparable ionic and electronic conductivity, both ionic and electronic thermopower have to be measured as a function of time. In other terms, the gradient of the chemical potential of the mobile component has to be measured by suitable chemical potential probes. Experiments on oxide materials, which are of particular interest in materials science, are difficult since the necessary condition of closed surfaces is hard to realize.

\section{Acknowledgments}

Financial support by the DFG (Deutsche Forschungsgemeinschaft) through the SFB 173 at the University of Hannover is gratefully acknowledged. The authors thank A.B. Lidiard (Oxford) and H. Schmalzried for critical reading of the manuscript and for valuable discussions and comments.

\section{References}

[1] S.R. de Groot and P. Mazur, Non-Equilibrium Thermodynamics (North-Holland, Amsterdam, 1962).

[2] C. Korte and J. Janek, submitted to J. Phys. Chem. Sol.

[3] C. Korte and J. Janek, to be published.

[4] S. Miyatani, J. Phys. Soc. Jpn. 24 (1968) 328.

[5] H. Rickert and C. Wagner, Ber. Bunsenges. Phys. Chem. 67 (1963) 621.
[6] J. Janek and C. Korte, Ber. Bunsenges. Phys. Chem. 99 (1995) 932.

[7] C. Korte and J. Janek, Ber. Bunsenges. Phys. Chem. 100 (1996) 425

[8] J.-W. Hahn, Solid State Ionics 81 (1995) 119.

[9] D.-L. Kim and H.-I. Yoo, Solid State lonics 81 (1995) 135.

[10] M. Kamata and T. Esaka, J. Appl. Electrochem. 24 (1994) 390.

[11] F. Millot and P. Gerdanian, J. Phys. Chem. Sol. 43 (1982) 501.

[12] F. Millot and P. Gerdanian, J. Nucl. Mater. 135 (1985) 197.

[13] H.I. Yoo and J.-H. Hwang, J. Phys. Chem. Sol. 53 (1992) 973.

[14] C. Wagnet, Progr. Solid State Chem. 7 (1972) 1.

[15] R.E. Howard, PhD Thesis (University of Oxford, Oxford, 1954).

[16] J. Janek and C. Korte, Z. Phys. Chem., in print.

[17] Gmelins Handbuch der Anorganischen Chemie, 8th ed. (Verlag Chemie, Weinheim, 1973) p. 30.

[18] CRC Handbook of Physics and Chemistry, 70th ed. (CRC Press, Boca Raton, 1990).

[19] H. Reye and H. Schmalzried, Z. Phys. Chem. N. F. 128 (1981) 93.

[20] K.D. Becker, H. Schmalzried und V. v. Wurmb, Solid State Jonics 11 (1983) 213.

[21] U. v. Oehsen and H. Schmalzried, Ber. Bunsenges. Phys. Chem. 85 (1981) 7.

[22] N. Valverde, Z. Phys. Chem. N. F. 70 (1970) 113.

[23] S. Miyatani, J. Phys. Soc. Jpn. 14 (1959) 996.

[24] J. Corish and P.W.M. Jacobs, J. Phys. Chem. Sol. 33 (1972) 1799.

[25] J. Mizusaki and K. Fueki, Rev. Chim. Miner. 17 (1980) 356.

[26] J.R. Haynes and W. Shockley, Phys. Rev. 82 (1951) 935.

[27] R.A. Smith, Semiconductors, 2nd ed. (Cambridge University Press, Cambridge, 1978) p. 151.

[28] I. Ebert and J. Teltow, Ann. Phys. 15 (1955) 268.

[29] R.W. Christy, J. Chem. Phys. 34 (1961) 1148.

[30] J. Corish and P.W.M. Jacobs, J. Phys. C: Solid State Phys. 6 (1973) 57

[31] Private communication.

[33] J.D. Hodge and H.K. Bowen, J. Am. Ceram. Soc. 64 (1981) $220 ; 64$ (1981) $431 ; 65$ (1982) 582 . 